\section{University of Leeds: impact of information literacy initiatives}

\author{
Helen Howard and \\ Martin Gill
}

\section{The Authors}

Helen Howard is Information Literacy Team Leader at the University of Leeds and Martin Gill is Faculty Team Leader (Social Sciences)

\section{Introduction}

At Leeds University Library, we decided to measure the impact of our work on Information Literacy, and in particular our IL online tutorials, with the aim of demonstrating that the time, effort and funding invested in this area of work made a real difference to library users.

We have a long history of involvement in Information Literacy, but felt that we needed more evidence of its impact on students to convince academics that it was worth embedding into their courses. This was a major aim of introducing an IL Strategy for the University, which was rolled out through the University's committee structure in 2003-2004. The IL Strategy outlines the need to incorporate IL into the mainstream curriculum and gained agreement from Learning and Teaching Board and Faculty Committees.

The introduction of the IL strategy tied in with the development of a number of online tutorials providing support for generic IL skills. The tutorial content had not been devised or written at the start of the IMPACT project, nor had the project officer been appointed, so IMPACT appeared to give a structure within which to evaluate their use and effectiveness. We hoped that the tutorials would provide examples of good quality online materials to share with academic staff, and that the IMPACT work would present information on their usage and impact on student learning.

In addition, Library staff were generally spending increasing amounts of time preparing and delivering IL sessions, so from a management viewpoint it was important to know that this was time well spent. There had also been some debate amongst Faculty Team (subject) Librarians over the usefulness of generic IL resources, so we were keen to demonstrate the value of the tutorials to all staff and encourage uptake of them.

In summary, therefore, we were aiming to embed IL further into the curriculum, through the introduction of an IL Strategy and the development of online tutorials. In addition, we hoped that the online tutorials would save staff time, by enabling them to use ready-made materials within teaching sessions where appropriate.

\section{Application of the Impact Implementation Initiative}

\section{Success Criteria}

We had 3 basic objectives which were: to improve student skills, to increase usage of the tutorials and to improve collaboration with academic staff. Our specific success criteria were:

- Increased use of e-resources by students

- Improvements in the quality and delivery of bibliographies in assessed work

- Increased use of document supply by students

- Improved understanding of search strategy techniques

- More students using IL tutorials

- More Schools using IL tutorials within courses

- More Faculty Team Librarians contributing to courses 
- Increased consultation / involvement in course planning stage

- Inclusion of IL in progress files

- Increased inclusion of IL as an integral part of courses

\section{Evidence Collected}

We tried to collect a range of evidence to assess to what extent we were meeting our success criteria. This included:

- Athens and Library Management system statistics (baseline and after) for a picture of e-resource usage

- Interviews with academics

- Pre- and post-training evaluation of student skills

- $20 \%$ sample of bibliographies (baseline and after) to assess improvement

- Evidence of reflection in student progress files

- Document supply statistics to assess the range of resources used

- Evaluation of search strategies produced by students

- Web statistics of online tutorial usage

- Survey of Faculty Team Librarians to assess extent of involvement in courses / course planning and usage of online tutorials in courses

- User evaluation of online tutorials (students and tutors)

- Assessment of existing Key Performance Indicators providing data of FTL involvement in courses

- Data on the number of committees where IL discussed

\section{Methods used}

A variety of quantitative methods were used for gathering data relating to the usage of eresources, online tutorials and document supply. These were often already available as existing statistics or key performance indicators which suited our purpose.

Pre and post-training evaluation questionnaires were used with groups of students to measure skills levels in areas of referencing and search techniques. A quiz was used to assess levels of understanding and learning amongst students completing the searching tutorial. This helped to establish the quality of the tutorial content, as well as the impact on student skills.

Basic questionnaire evaluation forms were provided to large numbers of students to assess the usability and usefulness of tutorials (as perceived by students). In addition, Faculty Team Librarians using the tutorials within a teaching session completed a tutor evaluation form to rate the success of this approach, as compared with a traditional teaching session.

All Faculty Team Librarians were surveyed in September 2004 to assess the effect that the IL Strategy and online tutorials had had on their involvement in teaching on courses and curriculum development.

Contact with academic staff occurred in a number of ways, including individual meetings and through Learning and Teaching committees, with the aim of assessing improvements in student skills, as well as levels of library involvement in courses / course planning. Indication of improvements to assessed work was also sought.

Whilst we were generally effective in gathering the evidence we set out to, there were a few areas where this proved more difficult than expected. For example, we wanted statistics on the levels of usage of certain e-resources, but found that due to changes in the way data was provided by our systems we were not able to compare results. We also made less progress than we had hoped with analysing bibliographies, given the time needed to organise and carry this out, and in measuring the impact of IL in student progress files.

\section{Significant results and how they have been used}

Prior to the IMPACT work, we had little experience of pre and post evaluation, but have found this particularly useful. An example of this was where Design students completed a questionnaire at the start of the training session to determine which tutorials they should complete. This experience illustrated the need to pilot questions on a smaller group of students first, but overall we found this to be effective, if 
only as a way of encouraging students to concentrate on the skills they needed most. Some groups of students have completed the same test at the start and end of a teaching session, in order to test levels of learning.

We have now used pre and post evaluation with a variety of groups and have built up confidence in using this method. This has made us reflect on our evaluation techniques more generally and encouraged us to move away from standard feedback forms to more in-depth assessments, and awareness of these new techniques has been disseminated across the Library. In general, we have found it has given an added dimension to our usual evaluation and testing mechanisms.

Before developing the online tutorials there was concern that generic e-learning tools would not be sufficiently relevant and tailored to support student learning, compared to subject-specific training. The IMPACT project work provided evidence that students can improve their skills using generic e-learning resources. The results have allowed us to use e-learning tools with more confidence and to develop them further. Now that the full set of tutorials is completed, we can launch them to academic staff with evidence of their usage and impact.

The tutorials have been thoroughly evaluated, and, as a result, revised and improved, thanks to the work undertaken as part of IMPACT. We have looked at the usability and usefulness for students, as well as from the tutor perspective. We have collected data that convinces us that students are comfortable using this format, with a very high proportion rating them easy to use and preferable to a paper workbook. In addition, by far the majority of students thought they would revisit the tutorials again, providing us with evidence that the development of further tutorials would not be wasted effort. We were also encouraged to see that correct responses to the quiz were provided by the majority of students, even where many had rated the tutorial content "all" or "mostly" new to them.

Evidence collected from Faculty Team Librarians shows that the majority of them have found an opportunity to use or promote the generic online tutorials, despite the initial conviction amongst many, that they could not provide a substitute to subject-specific training. We have seen a significant rise in the use of IL packages in Semester 1 2004-2005, satisfying our second overall objective. We have also seen increased involvement and collaboration with academic staff in a number of ways. This has been mainly due to the roll-out of our IL Strategy and, again, evidence gathered as part of the IMPACT work enables us to show the difference IL can make.

\section{What has been learnt from participation}

In general, participation in the IMPACT project gave us a structured methodology with which to evaluate a key service. It provided the opportunity to plan the project outline away from base, enabling us to think "outside the box" and come up with a range of criteria which we might otherwise have missed. We were, however, slightly over-optimistic in terms of timescales and the overall scope of the project, particularly as the service being evaluated had yet to be developed. On reflection, it proved difficult to develop a new service and try to evaluate it at the same time, though overall the findings have helped to develop the service further and promote it more widely. 